\title{
Erratum to: Probing and tracking organelles in living plant cells
}

\author{
Tong Chen • Xiaohua Wang • Daniel von Wangenheim • \\ Maozhong Zheng • Jozef Šamaj • Wanquan Ji • \\ Jinxing Lin
}

Published online: 4 February 2012

(C) Springer-Verlag 2012

\section{Erratum to: Protoplasma}

\section{DOI: 10.1007/s00709-011-0364-4}

The affiliation of Jozef Šamaj unfortunately contains a mistake. It should have been,

Centre of the Region Haná for Biotechnological and Agricultural Research, Department of Cell Biology, Faculty of Science, Palacký University Olomouc,

Slechtitelu 11, 78371 Olomouc, Czech Republic
The online version of the original article can be found at http://dx.doi. org/10.1007/s00709-011-0364-4.

T. Chen $\cdot$ X. Wang $\cdot$ M. Zheng $\cdot$ J. Lin

Key Laboratory of Plant Molecular Physiology, Institute of Botany, Chinese Academy of Sciences,

Beijing 100093, China

D. von Wangenheim

Institute of Cellular and Molecular Botany,

University of Bonn,

Kirschallee 1,

53115 Bonn, Germany

\section{J. Šamaj}

Institute of Plant Genetics and Biotechnology,

Slovak Academy of Sciences,

Akademicka 2,

SK-95007 Nitra, Slovak Republic
J. Šamaj

Centre of the Region Haná for Biotechnological and Agricultural

Research, Department of Cell Biology, Faculty of Science,

Palacký University Olomouc,

Slechtitelu 11,

78371 Olomouc, Czech Republic

W. Ji

College of Agriculture,

Northwest Sci-Tech University of Agriculture and Forestry,

Yangling, Shanxi, China

J. Lin $(\triangle)$

Institute of Botany, Chinese Academy of Sciences,

Xiangshan, Beijing 100093, China

e-mail: linjx@ibcas.ac.cn 